\title{
Genome-wide association study of early-onset bipolar I disorder in the Han Taiwanese population
}

\author{
Lawrence Shih-Hsin Wu', Ming-Chyi Huang ${ }^{2,3}$, Cathy Shen-Jang Fann ${ }^{4}$, Hsien-Yuan Lane ${ }^{1,5}$, Chian-Jue Kuo ${ }^{2,3}$, \\ Wei-Che Chiu (1) ${ }^{6,7}$, Pui-Yan Kwok ${ }^{4}$ and Andrew Tai-Ann Cheng (10)
}

\begin{abstract}
The search for susceptibility genes underlying the heterogeneous bipolar disorder has been inconclusive, often with irreproducible results. There is a hope that narrowing the phenotypes will increase the power of genetic analysis. Earlyonset bipolar disorder is thought to be a genetically homogeneous subtype with greater symptom severity. We conducted a genome-wide association study (GWAS) for this subtype in bipolar I (BPI) disorder. Study participants included 1779 patients of Han Chinese descent with BPI disorder recruited by the Taiwan Bipolar Consortium. We conducted phenotype assessment using the Chinese version of the Schedules for Clinical Assessment in Neuropsychiatry and prepared a life chart with graphic depiction of lifetime clinical course for each of the BPI patient recruited. The assessment of onset age was based on this life chart with early onset defined as $\leq 20$ years of age. We performed GWAS in a discovery group of 516 early-onset and 790 non-early-onset BPI patients, followed by a replication study in an independent group of 153 early-onset and 320 non-early-onset BPI patients and a meta-analysis with these two groups. The SNP rs11127876, located in the intron of CADM2, showed association with early-onset BPI in the discovery cohort $\left(P=7.04 \times 10^{-8}\right)$ and in the test of replication $(P=0.0354)$. After meta-analysis, this SNP was demonstrated to be a new genetic locus in CADM2 gene associated with early-onset BPI disorder $\left(P=5.19 \times 10^{-8}\right)$.
\end{abstract}

\section{Introduction}

Bipolar disorder (BPD) is a severe, chronic, and disabling mental illness characterized by recurrent episodes of hypomania or mania and depression ${ }^{1,2}$. It is a clinically defined nosological entity with multifactorial but poorly understood etiologic mechanisms. The evidence from twin, family, and adoption studies provide compelling evidence for a strong genetic predisposition to $\mathrm{BPD}^{3}$ with heritability estimated to be as high as $\geq 80 \%^{3-5}$.

Given BPD is a heterogeneous disease with substantial phenotypic and genetic complexities ${ }^{6}$, the identification for BPD risk loci has proven to be difficult. Some researchers have proposed that dissecting BPD into

Correspondence: Andrew Tai-Ann Cheng (bmandrew@gate.sinica.edu.tw)

${ }^{1}$ Graduate Institute of Biomedical Sciences, China Medical University, Taichung, Taiwan

${ }^{2}$ Department of Psychiatry, Taipei City Psychiatric Center, Taipei City Hospital, Taipei, Taiwan

Full list of author information is available at the end of the article clinical subgroups with distinct sub-phenotypes may result in genetically homogeneous cohorts to facilitate the mapping of BPD susceptibility genes ${ }^{6}$. Among the subphenotypes, early-onset BPD is of particular interest as several independent cohort studies have demonstrated their existence ${ }^{7-11}$.

Comparing to the non-early-onset BPD, the early-onset subtype is associated with a more severe form of clinical manifestations characterized by frequent psychotic features, more mixed episodes, greater psychiatric comorbidity such as drug and alcohol abuse and anxiety disorders ${ }^{12,13}$, higher risk of suicide attempt ${ }^{14}$, worse cognitive performance ${ }^{15}$, and poorer response to prophylactic lithium treatment ${ }^{13,16-20}$. In addition, the pattern of disease inheritance seems to differ between earlyand late-onset BPD families ${ }^{21,22}$, with the former involving greater heritability ${ }^{23}$. These observations indicate that early-onset BPD may be a genetically homogenous

\section{(c) The Author(s) 2021}

(c) (i) Open Access This article is licensed under a Creative Commons Attribution 4.0 International License, which permits use, sharing, adaptation, distribution and reproduction cc) in any medium or format, as long as you give appropriate credit to the original author(s) and the source, provide a link to the Creative Commons license, and indicate if changes were made. The images or other third party material in this article are included in the article's Creative Commons license, unless indicated otherwise in a credit line to the material. If material is not included in the article's Creative Commons license and your intended use is not permitted by statutory regulation or exceeds the permitted use, you will need to obtain permission directly from the copyright holder. To view a copy of this license, visit http://creativecommons.org/licenses/by/4.0/. 
subset and thus could be used for genetic study to identify its susceptibility genes.

A number of BPD genes identified by genome-wide association study (GWAS) have been widely replicated and intensively studied (e.g., CACAN1C and $A N K 3)^{24}$, but these studies did not include early-onset BPD. Over the past two decades, a host of studies have investigated genetic loci responsible for early-onset BPD through linkage-analyses, candidate-gene association, analyses of copy number variants (CNVs), and GWAS, but findings are inconclusive. Candidate-gene association studies have identified a number of genes potentially associated with early-onset BPD, including glycogen synthase kinase 3- $\beta$ gene $^{25}$, circadian clock gene Per $3^{26}$, serotonin transporter gene $^{27}$, brain-derived neurotrophic factor gene $^{28}$, and gene coding synaptosomal-associated protein SNAP2 $25^{29}$. However, very few positive findings of these studies have been replicated independently. Findings from linkage studies suggested chromosomal regions harboring the susceptibility genes at $3 \mathrm{p} 14$ and $21 \mathrm{q} 22$, plus the loci at 18p11, 6q25, 9q34 and 20q11 with nominal significance $^{21,30,31}$. Studies of CNVs in early-onset BPD were based on relatively small effect sizes and were irreproducible, suggesting that CNVs are unlikely the major source of liability ${ }^{32-35}$. Finally, GWAS failed to find any risk variant with genome-wide statistical significance in Caucasian populations, despite some variants showed suggestive significance ${ }^{36}$.

In previous genetic studies, the definition of early-onset in BPD typically ranged from 15 to 25 years of age. These association studies were largely conducted with small sample size and were underpowered $(<80 \%)^{36,37}$. Most of them (including GWAS) compared early-onset BPD vs. healthy control. Such a case-control design is more likely to identify susceptibility gene(s) for BPD per se, but not for the early-onset subtype. The optimal strategy to identify gene(s) for the early-onset BPD is to include the non-early-onset BPD group for comparison. Different definitions for early onset of BPI have been proposed in previous work. In this paper, we reported findings from a GWAS with high-density SNP chips on early-onset, defined as $\leq 20$ years of age, BPI patients of Han Taiwanese descent.

\section{Materials and methods}

\section{Study participants}

Study participants included a total of 1779 unrelated Han Taiwanese BPI patients recruited from psychiatric departments of general hospitals and psychiatric institutions in the Taiwan Bipolar Consortium ${ }^{38,39}$.

\section{Phenotype definition and assessment}

The clinical phenotype assessment of manic and depressive episodes was performed by well-trained psychiatric nurses and psychiatrists using a crossculturally validated and reliable Chinese version of the Schedules for Clinical Assessment in Neuropsychiatry ${ }^{40}$, supplemented by available medical records. All of them were diagnosed according to the DSM-IV criteria for BPI disorder with recurrent episodes of mania with or without depressive episode(s).

The assessment of onset age was based on a life chart prepared with graphic depiction of lifetime clinical course for each of the BPI patient recruited ${ }^{41}$. This life chart included largely all the mood episodes with date of onset (year and month), duration, and severity (including the extent of functional disability, hospitalization, and the presence of psychotic features). The construction of this life chart was based on integrated information gathered from direct interview with patients and their family members, interviews with in-charge psychiatrists, and a thorough medical chart review.

Different definitions for early onset of BPI have been proposed in previous work. Our selection of 20 as the age threshold was based on a systematic review for pediatric $\mathrm{BPD}^{42}$. The age at onset was defined by the first mood episode (depressive, manic, hypomanic, or mixed). Of all patients, 1306 (516 with onset age $\leq 20$ and $790>20$ ) with genotyping data available were included in the discovery group for GWAS and the rest 473 (153 with onset age $\leq 20$ and $320>20$ ) without genotyping data were included in the replication group.

\section{Genotyping}

Genotyping was performed using the Illumina HumanOmni1-Quad BeadChip $(N=936)$ and the HumanOmni2.5-Quad BeadChip $(N=575)$ by Chun-Tai Co. (Taipei, Taiwan). For this study, we integrated the two genome-wide SNP data sets through imputation with 1000 Genomes. To bridge the two sets, we also genotyped 82 of the first 936 participants using the HumanOmni2.5Quad BeadChip. The two gene chips shared about $750 \mathrm{~K}$ common SNPs; thus, we can check the genotyping consistency of the two sets. Genotype calling for the two data sets was determined by BeadStudio (Illumina) using default parameters. The genotype imputation method, IMPUTE $2^{43}$, was performed under default setting to estimate the genotypes of SNPs not on array. In the imputation process, reference haplotypes was curated from 1000 Genomes Project Phase III $^{44}$. To improve the efficiency, we performed a whole genome imputation in every $5 \mathrm{Mb}$ chunk, respectively. ANNOVAR ${ }^{45}$ was used to annotate the functional consequences of singlenucleotide variants found in our data set. The imputation data set included genotyping information from 1429 $(936+575-82)$ patients and 1306 of them with clear onset-age data were included in the discovery group for GWAS. 
The subsequent quality control of the genotype data was implemented for each data set to exclude the following SNPs and individuals before the imputation: (1) individuals with a call rate $<98 \%$; (2) $P<1.0 \times 10^{-4}$ for Hardy-Weinberg violation; (3) SNPs with MAF < 5\%; (4) samples with first-degree cryptic relationships; and (5) samples that were potentially contaminated.

We used the Agena MassARRAY platform with iPLEX chemistry (Agena, San Diego, CA) for replication and confirmed the genotyping result from SNP arrays. The Spectro-CHIPs were analyzed using the MassARRAY Analyzer 4, and the results were analyzed using clustering analysis with the TYPER 4.0 software. The allele-specific diagnostic products had a unique molecular weight that were identified using matrix-assisted laser desorption ionization time-of-flight mass spectrometry.

\section{Statistical analysis}

A principal component analysis (PCA) with 1306 patients in discovery group based on the genome-wide IBS (identical by state) pairwise distances was performed using PLINK v. 1.9 (https://www.cog-genomics.org/ plink2) $)^{46}$. GWAS was carried out for the discovery group, by comparing allele frequencies between earlyonset $(\leq 20)$ and non-early-onset $(>20)$ BPI patients. The threshold $P$ value was set at $1.05 \times 10^{-8}$ after a Bonferroni correction for the number of SNPs $(4,750,978$, including imputed SNPs). Because few SNPs reached the significance after Bonferroni correction, top SNPs with $P$ values less than $10^{-7}$ were also considered for further examinations. Quantile-quantile $(\mathrm{Q}-\mathrm{Q})$ plots were then used to examine the $P$ value distributions. The calculation of GWAS and Q-Q plots was performed using PLINK v. $1.9^{46}$. Top SNPs identified in the GWAS were replicated with 473 patients in the replication group. A metaanalysis was then performed by SAS 9.4 (SAS Institute Inc., Cary, NC, USA), using its existing macros. We adopted the macro as " $x=-2 \times(\log ($ GWAS $P$ value $)+\log$ (replication $P$ value)), metaP $=1-\mathrm{CDF}$ ('CHISQUARE', $x, 4)$ " to calculate the $P$ value, where CDF represents cumulative distribution function. We ran meta-analysis using SAS existing macro based on $P$ values from GWAS and replication. The meta-analysis was also performed by PLINK v. $1.9^{46}$ based on the odds ratio (OR), standard error of OR, and $P$ values.

\section{Results}

Demographic and clinical characteristics of the patients in the discovery $(n=1306)$ and the replication $(n=473)$ groups are shown in Table 1 . Female preponderance was observed in both groups and in all early- and non-early-onset subgroups, but the difference was not statistically significant in all of them. Rates of psychotic features and suicide attempt were higher in early-onset subgroups than in their non-early-onset counterparts in both discovery and replication groups (90.8\% versus $84.2 \%$ and $90.2 \%$ versus $80.9 \%$ for psychotic features; $51.1 \%$ versus $43.7 \%$ and $41.8 \%$ versus $34.3 \%$ for suicide attempt, respectively). A higher proportion of the first mood episode was found to be depression in the early-onset subgroup in both discovery and replication groups $(45.2 \%$ and $39.9 \%$, respectively). The difference was statistically significant in the discovery group $\left(\chi^{2}=7.9117, P=0.005\right)$.

Table 1 Demographic and clinical characteristics of study patients with bipolar I (BPI) disorder ${ }^{\mathrm{a}}$.

\begin{tabular}{|c|c|c|c|c|c|c|}
\hline & \multicolumn{3}{|c|}{ Discovery group } & \multicolumn{3}{|c|}{ Replication group } \\
\hline & $\begin{array}{l}\text { Onset } \leq 20 \\
(N=516)\end{array}$ & $\begin{array}{l}\text { Onset }>20 \\
(N=790)\end{array}$ & $P$ value & $\begin{array}{l}\text { Onset } \leq 20 \\
(N=153)\end{array}$ & $\begin{array}{l}\text { Onset }>20 \\
(N=320)\end{array}$ & $P$ value \\
\hline Sex & & & 0.843 & & & 0.938 \\
\hline Male & $229(44.4 \%)$ & $355(44.9 \%)$ & & $74(48.4 \%)$ & $156(48.7 \%)$ & \\
\hline Female & $287(55.6 \%)$ & $435(55.1 \%)$ & & $79(51.6 \%)$ & $164(51.3 \%)$ & \\
\hline Age at onset & $17.02 \pm 2.54$ & $31.83 \pm 9.29$ & $<0.001$ & $17.65 \pm 1.99$ & $32.05 \pm 9.03$ & $<0.001$ \\
\hline Age at recruitment & $37.56 \pm 11.79$ & $41.84 \pm 11.63$ & $<0.001$ & $37.56 \pm 12.52$ & $47.23 \pm 12.35$ & $<0.001$ \\
\hline With psychotic features (\%) & $465(90.8 \%)$ & $665(84.2 \%)$ & 0.002 & $138(90.2 \%)$ & $259(80.9 \%)$ & 0.010 \\
\hline With suicide attempt & $264(51.1 \%)$ & $345(43.7 \%)$ & 0.008 & $64(41.8 \%)$ & $110(34.3 \%)$ & 0.116 \\
\hline First mood episode & & & 0.005 & & & 0.685 \\
\hline Mania & $283(54.8 \%)$ & $495(62.7 \%)$ & & $92(60.1 \%)$ & $205(64.1 \%)$ & \\
\hline Depression & $233(45.2 \%)$ & 295 (37.3\%) & & 61 (39.9\%) & 115 (35.9\%) & \\
\hline
\end{tabular}

ancluding early-onset $(\leq 20)$ and non-early-onset $(>20)$ patients in both discovery and replication groups. 


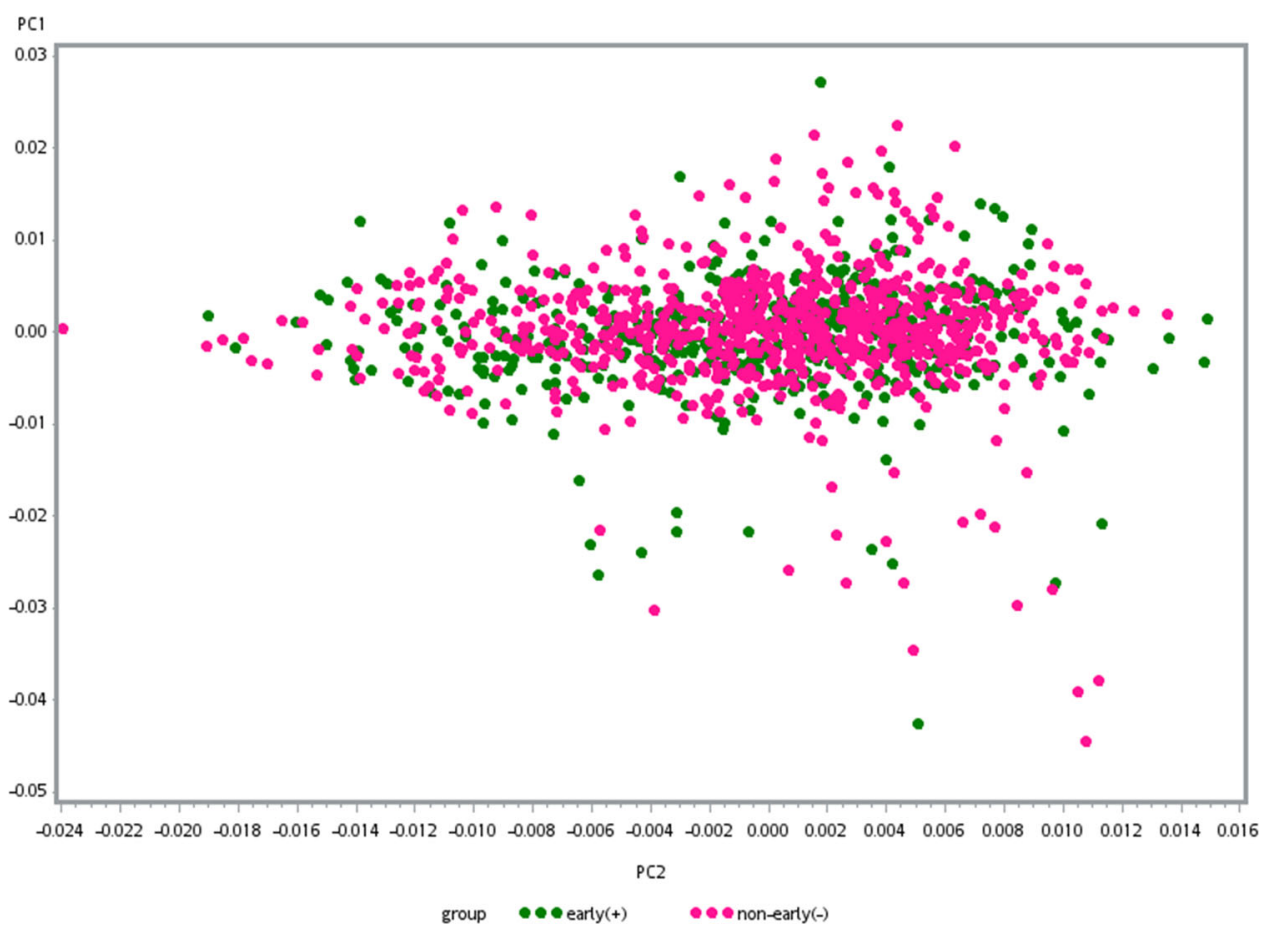

Fig. 1 The principal component analysis plot of the 1306 GWAS samples. The $Y$ and $X$ axes are the first and second dimensions from principal component analysis based on the genome-wide IBS pairwise distances among the 1306 GWAS subjects. Green crosses represented early-onset and pink non-early-onset BPI. The two axes correspond to a reduced representation of 10,000 randomly selected SNPs into two dimensions. No clustering pattern was found, indicating that neither substantial population stratification nor cryptic relationship among the 1306 subjects was found.

\section{Association analysis}

Genotyping consistency of the common SNPs in the two sets (HumanOmni1-Quad BeadChip and HumanOmni2.5-Quad BeadChip) was checked in 82 sample and the average genotyping consistency of $>99.98 \%$ was found. The PCA was used to present the genotyping consistency (Supplementary Fig. 1). PCA did not find substantial population stratification and cryptic relationship among the 1306 GWAS subjects (Fig. 1). The PCA was also performed to check whether there is a sampling bias between the two chips. No stratification and cryptic relationship was found between subjects with each of them (Supplementary Fig. 2). Figure 2 shows the $\mathrm{Q}-\mathrm{Q}$ plot from results of the GWAS for early-onset BPI and the lambda value was 1.067. Associations between individual SNPs and early onset in 1306 patients with BPI calculated by $\chi^{2}(1 \mathrm{df})$ for basic allelic test was plotted against the chromosomal location across the genome (Fig. 3). The horizontal line indicates the genome-wide significance level of $P=1.05 \times 10^{-8}$, achieved by one SNP located on upstream of MIR522. In addition, three SNPs reached nominal significance at $1.05 \times 10^{-8}<P<10^{-7}$. They were located in the intron of CADM2 (cell adhesion molecule 2) and $C U B N$ (cubilin), and in intergenic region between MFHAS1 (malignant fibrous histiocytoma amplified

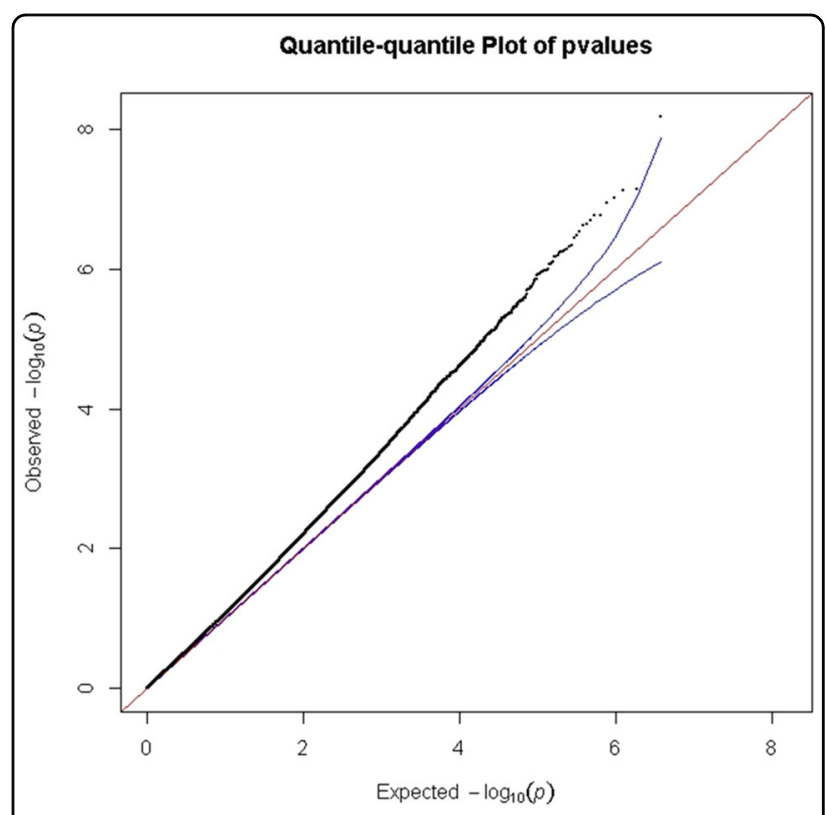

Fig. $2 \mathrm{Q}-\mathrm{Q}$ plot of the Fisher's test for allelic model $P$ values in the discovery group of BPI disorder $(N=1306)$. The upper and lower boundaries of the $95 \%$ confidence bands are represented by the blue lines. 


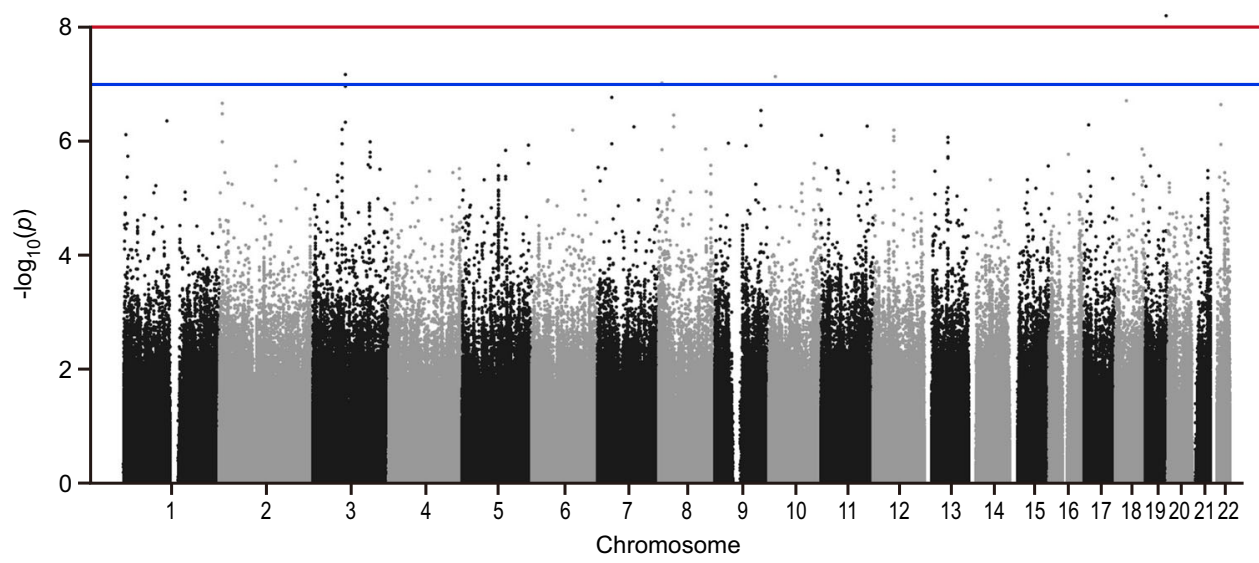

Fig. 3 Genome-wide association between single-nucleotide polymorphisms (SNPs) and early-onset BPI disorder in the discovery group. The negative log of the $P$ value for the association for allelic model was plotted against the chromosomal location across the genome. The upper and lower horizontal lines indicate the genome-wide significance level of $1.05 \times 10^{-8}$ and $10^{-7}$, respectively.

Table 2 Distribution of allele frequency and $P$ value of top four SNPs significantly associated with early-onset BPI disorder ( $\leq 20$ years of age) in discovery and replication groups.

\begin{tabular}{|c|c|c|c|c|c|c|c|c|c|c|}
\hline \multirow[t]{2}{*}{ SNP ID } & \multirow[t]{2}{*}{ CHR } & \multirow[t]{2}{*}{$\mathrm{BP}^{\mathrm{a}}$} & \multirow[t]{2}{*}{ Gene/Feature } & \multirow[t]{2}{*}{ Allele } & \multirow[t]{2}{*}{ Group } & \multicolumn{2}{|c|}{$\begin{array}{l}\text { Minor allele } \\
\text { frequency (\%) }\end{array}$} & \multirow[t]{2}{*}{$P$} & \multirow[t]{2}{*}{ OR $(95 \% \mathrm{Cl})$} & \multirow[t]{2}{*}{$\begin{array}{l}P_{\text {meta(SAS)/ }} \\
P_{\text {meta(PLINK) }}\end{array}$} \\
\hline & & & & & & $\leq 20$ & $>20$ & & & \\
\hline \multirow[t]{2}{*}{ rs75928006 } & 19 & 54254388 & MIR522/upstream & $C / T$ & Discovery & 9.4 & 3.9 & $6.45 \times 10^{-9}$ & $2.58(1.86,3.60)$ & $9.23 \times 10^{-8} / 8.14 \times 10^{-5}$ \\
\hline & & & & & Replication & 15.7 & 16.7 & 0.7084 & $0.93(0.64,1.34)$ & \\
\hline \multirow[t]{2}{*}{ rs11127876 } & 3 & 85124697 & CADM2/intronic & $C / T$ & Discovery & 9.3 & 4.1 & $7.04 \times 10^{-8}$ & $2.39(1.73,3.31)$ & $5.19 \times 10^{-8} / 1.26 \times 10^{-7}$ \\
\hline & & & & & Replication & 21.2 & 15.6 & 0.0354 & $1.46(1.03,2.06)$ & \\
\hline \multirow[t]{2}{*}{ rs7914868 } & 10 & 16973722 & CUBN/intronic & $C / G$ & Discovery & 12.4 & 6.3 & $7.59 \times 10^{-8}$ & $2.10(1.59,2.76)$ & $7.00 \times 10^{-7} / 8.31 \times 10^{-6}$ \\
\hline & & & & & Replication & 24.5 & 22.5 & 0.5102 & $1.12(0.81,1.54)$ & \\
\hline \multirow[t]{2}{*}{ rs10106565 } & 8 & 8855032 & MFHAS1-ER/1/intergenic & $\mathrm{C} / \mathrm{T}$ & Discovery & 9.8 & 4.5 & $9.70 \times 10^{-8}$ & $2.31(1.68,3.16)$ & $1.33 \times 10^{-7} / 4.38 \times 10^{-5}$ \\
\hline & & & & & Replication & 18.6 & 17.8 & 0.7866 & $1.06(0.74,1.50)$ & \\
\hline
\end{tabular}

$P$ values, $\mathrm{ORs}$, and $\mathrm{Cls}$ were calculated based on the allele model.

$O R$ odds ratio, $C l$ confidence interval, $P_{\text {meta }}(S A S), P_{\text {meta (PLINK) }} P$ value of meta-analysis generated by SAS and PLINK v. 1.9 program.

aLocation was cited according to GRCh37.p13.

sequence 1) and ERI1 (exoribonuclease 1) (Table 2), respectively.

These four top SNPs were then genotyped in the replication group. The SNP rs11127876, located in the intron of $C A D M 2$, showed nominal association $(P=$ 0.0354, not resist to correction for multiple testing) (Table 2). The association in GWAS (discovery group) of SNPs in $C A D M 2$ genomic region was further checked and some SNPs near rs11127876 also showed nominal association (Supplementary Fig. 3). We finally performed metaanalysis for both discovery and replication groups. The SNP rs11127876 showed the top association in both approaches $\left(P=5.19 \times 10^{-8}\right.$ using SAS; $P=1.26 \times 10^{-7}$ using PLINK) close to genome-wide significance.

\section{Discussion}

In this paper, we have reported one of the largest GWASs to investigate genetic susceptibility to early-onset BPI with the first mood episode occurring $\leq 20$ years of age. We employed standardized psychiatric interview and constructed a life chart with detailed clinical history to ensure the accuracy and homogeneity of phenotype for genotyping. Our GWAS with high-density SNP chips identified the SNP rs11127876 in CADM2 gene to be 
associated with early-onset BPI in both discovery and replication groups, and meta-analysis for the association was close to genome-wide significance $\left(P=5.19 \times 10^{-8}\right)$.

The gene CADM2 on chromosome 3 encodes a synaptic cell adhesion molecule that is prominently expressed in neurons, and plays key roles in the development, differentiation, and maintaining synaptic circuitry of the central nervous system ${ }^{47}$. In previous GWASs, CADM2 has been found to be associated with a number of mental healthrelated traits, including alcohol consumption ${ }^{48}$, cannabis use $^{49,50}$, reduced anxiety, neuroticism and conscientiousness, and increased risk-taking behavior ${ }^{51,52}$. CADM2 was also reported to be associated with executive functioning and processing speed $^{53}$, general cognitive function ${ }^{54}$, and educational attainment ${ }^{55}$.

Though there have been no investigations examining the risk-taking phenotype in early-onset relative to nonearly-onset BPD, Homes et al. showed that BPD patients with a past history of alcohol abuse or dependence had a higher risk-taking propensity ${ }^{56}$, suggesting a relationship between early-onset BPD and risk-taking propensity.

Of note, Morris et al. suggested that CADM2 variants may not only link with psychological traits, but also influence metabolic-related traits, such as body mass index, blood pressure, and C-reactive protein ${ }^{57}$. In addition, they found that $C A D M 2$ variants had genotypespecific effects on CADM2 expression levels in adult brain and adipose tissues. The finding highlights the potential pleiotropy of $C A D M 2$ gene, i.e., the genetic variants may influence multiple traits, and shared biological mechanisms across brain and adipose tissues through the regulation of CADM2 expression ${ }^{57}$. Given that the metabolic comorbidities are prevalent in patients with early-onset $\mathrm{BPD}^{58}$, it is conceivable that $C A D M 2$ variants may influence both psychological (emotional processing) and physical (metabolic regulation) traits, further contributing to a more severe clinical subtype of BPD with accompanying risk of metabolic adversities. In addition, an association between risk-taking and obesity has also been implicated in previous research, which suggests that risktakers tend to overlook health-related outcomes and are prone to aberrant reward circuitry predisposing them to poor dietary choices and excessive intake $\mathrm{k}^{57,59}$.

Collectively, in line with the characteristics found to be associated with $C A D M 2$ variants, it is likely that $C A D M 2$ may exert an effect on the constellation of clinical features related to early-onset BPD with greater symptom severity (Table 1). Therefore, our findings suggest that CADM2 genetic variants may have significant effects on a subtype of BPD with early-onset.

Two previous GWASs comparing early-onset BPD patients with healthy controls did not find any genetic variants reaching genome-wide significance ${ }^{36,37}$. Our study included a larger sample of early-onset BPI patients
$(N=669)$ to conduct GWAS using high-density genotyping $(4,750,978$ SNPs generated by gene chips and imputation). The statistical power was calculated using Post-hoc Power Calculator (https://clincalc.com/stats/ Power.aspx), combining the allelic frequencies of both discovery and replication groups. In this study of two independent samples of BPI with dichotomous endpoint, the power reached $99.4 \%$ and $18.2 \%$ under type I error $(\alpha)$ $=0.05$ and $=5 \times 10^{-8}$, respectively. Results of our study are also likely to be underpowered under the stringency setting of type I error. However, the frequency of risk allele $\mathrm{T}$ was higher in patients with onset $\leq 20$ than in patients with onset $>20$ in both discovery and replication groups. We believe all these have provided strong evidence to confirm the association of this SNP with earlyonset BPD.

In Table 2, the minor allele frequencies differ quite a bit between the discovery and replication cohorts. In the NCBI SNP database, minor allele frequency of rs 11127876 is $0.08(\mathrm{~T})$ in Han Chinese in Beijing, close to our results and suggest that the different allele frequencies observed in Table 2 may mainly result from our sampling. The over-representative minor allele frequency in replication group may have come from random sampling or effects of hidden characters (phenotypes) of our patients recruited. Genetic variant of CADM2 has been reported to be associated with behavioral and metabolic traits ${ }^{57}$, which were not assessed in this study. Though the minor allele frequencies of rs11127876 were different in discovery and replication groups, the same direction of ORs of rs11127876 minor allele supports the reliability of our findings.

The SNP rs75928006 located in the upstream of MIR522 reached genome-wide significance in discovery group but failed to show statistical significance in replication group. MIR522 promotes glioblastoma cell proliferation $^{60}$, but there was no evidence to suggest its association with any psychiatric disorders.

One major limitation of this study is the possibility of recall bias about the exact onset age of the first mood episode of BPI, particular when there was a long history of the illness. Previous studies have however suggested that age at onset can be assessed reliably ${ }^{61}$. The preparation of life chart containing detailed clinical course and treatment based on a semi-structured clinical interview plus a thorough medical chart review for individual patients should have overcome this potential limitation satisfactorily.

In summary, we have identified a genetic locus rs11127876 in CADM2 gene to be associated with earlyonset BPI. The finding has reflected the co-sharing genetic features of psychiatric disorders and behavioral traits. Further investigations of the CADM2 biological function in BPI are warranted. 


\section{Acknowledgements}

This study was supported by grants from Academia Sinica (AS 23-23, 52102310023C), Ministry of Science and Technology (106-2314-B-532-005MY3), China Medical University (CMU107-Z-06, CMU108-Z-09), and Taipei City Hospital (TPCH 108-057 and 109-066), Taiwan.

\section{Author details}

'Graduate Institute of Biomedical Sciences, China Medical University, Taichung, Taiwan. ${ }^{2}$ Department of Psychiatry, Taipei City Psychiatric Center, Taipei City Hospital, Taipei, Taiwan. ${ }^{3}$ Department of Psychiatry, School of Medicine, College of Medicine, Taipei Medical University, Taipei, Taiwan. ${ }^{4}$ Institute of Biomedical Sciences, Academia Sinica, Taipei, Taiwan. ${ }^{5}$ Department of Psychiatry, China Medical University Hospital, Taichung, Taiwan. ${ }^{6}$ Department of Psychiatry, Cathay General Hospital, Taipei 10630, Taiwan. ${ }^{7}$ School of Medicine, Fu Jen Catholic University, Taipei 24205, Taiwan

\section{Conflict of interest}

The authors declare no competing interests.

\section{Publisher's note}

Springer Nature remains neutral with regard to jurisdictional claims in published maps and institutional affiliations.

Supplementary information The online version contains supplementary material available at https://doi.org/10.1038/s41398-021-01407-6.

Received: 3 September 2020 Revised: 5 April 2021 Accepted: 21 April 2021 Published online: 20 May 2021

\section{References}

1. Frye, M. A. Clinical practice. Bipolar disorder-a focus on depression. N. Engl. J. Med. 364, 51-59 (2011).

2. Geddes, J. R. \& Miklowitz, D. J. Treatment of bipolar disorder. Lancet $\mathbf{3 8 1}$ 1672-1682 (2013).

3. Smoller, J. W. \& Finn, C. T. Family, twin, and adoption studies of bipolar disorder. Am. J. Med. Genet. C. Semin. Med. Genet. 123C, 48-58 (2003).

4. Burmeister, M., Mclnnis, M. G. \& Zöllner, S. Psychiatric genetics: progress amid controversy. Nat. Rev. Genet. 9, 527-540 (2008).

5. McGuffin, P. et al. The heritability of bipolar affective disorder and the genetic relationship to unipolar depression. Arch. Gen. Psychiatry 60, 497-502 (2003).

6. Leboyer, M., Henry, C., Paillere-Martinot, M. L. \& Bellivier, F. Age at onset in bipolar affective disorders: a review. Bipolar Disord. 7, 111-118 (2005).

7. Bellivier, F., Golmard, J. L., Henry, C., Leboyer, M. \& Schürhoff, F. Admixture analysis of age at onset in bipolar I affective disorder. Arch. Gen. Psychiatry $\mathbf{5 8}$ 510-512 (2001).

8. Bellivier, F. et al. Age at onset in bipolar I affective disorder: further evidence for three subgroups. Am. J. Psychiatry 160, 999-1001 (2003).

9. Hamshere, M. L. et al. Age-at-onset in bipolar-l disorder: mixture analysis of 1369 cases identifies three distinct clinical sub-groups. J. Affect. Disord. 116, 23-29 (2009).

10. Lin, P. I. et al. Clinical correlates and familial aggregation of age at onset in bipolar disorder. Am. J. Psychiatry 163, 240-246 (2006).

11. Manchia, M. et al. Age at onset in Sardinian bipolar I patients: evidence for three subgroups. Bipolar Disord. 10, 443-446 (2008).

12. McElroy, S. L. et al. Axis I psychiatric comorbidity and its relationship to historical illness variables in 288 patients with bipolar disorder. Am. J. Psychiatry 158, 420-426 (2001).

13. Schürhoff, F. et al. Early and late onset bipolar disorders: two different forms of manic-depressive illness? J. Affect. Disord. 58, 215-221 (2000).

14. Tsai, S. Y., Lee, J. C. \& Chen, C. C. Characteristics and psychosocial problems of patients with bipolar disorder at high risk for suicide attempt. J. Affect. Disord. 52, 145-152 (1999).

15. Tsai, S. Y., Lee, H. C., Chen, C. C. \& Huang, Y. L. Cognitive impairment in later life in patients with early-onset bipolar disorder. Bipolar Disord. 9, 868-875 (2007).

16. Craney, J. L. \& Geller, B. A prepubertal and early adolescent bipolar disorder-I phenotype: review of phenomenology and longitudinal course. Bipolar Disord. 5, 243-256 (2003)
17. Ernst, C. L. \& Goldberg, J. F. Clinical features related to age at onset in bipolar disorder. J. Affect. Disord. 82, 21-27 (2004).

18. Geller, B., Tillman, R., Bolhofner, K. \& Zimerman, B. Child bipolar I disorder: prospective continuity with adult bipolar I disorder; characteristics of second and third episodes; predictors of 8-year outcome. Arch. Gen. Psychiatry 65, 1125-1133 (2008).

19. Perlis, R. H. et al. Long-term implications of early onset in bipolar disorder: data from the first 1000 participants in the systematic treatment enhancement program for bipolar disorder (STEP-BD). Biol. Psychiatry 55, 875-881 (2004).

20. Post, R. M. et al. Early-onset bipolar disorder and treatment delay are risk factors for poor outcome in adulthood. J. Clin. Psychiatry 71, 864-872 (2010).

21. Etain, B. et al. Genome-wide scan for genes involved in bipolar affective disorder in 70 European families ascertained through a bipolar type I earlyonset proband: supportive evidence for linkage at 3p14. Mol. Psychiatry 11, 685-694 (2006).

22. Grigoroiu-Serbanescu, M. et al. Different familial transmission patterns in bipolar I disorder with onset before and after age 25. Am. J. Med. Genet. 105, 765-773 (2001)

23. Faraone, S. V. \& Tsuang, M. T. Heterogeneity and the genetics of bipolar disorder. Am. J. Med. Genet. C. Semin. Med. Genet. 123C 1-9 (2003).

24. Zhang, C., Xiao, X., Li, T. \& Li, M. Translational genomics and beyond in bipolar disorder. Mol. Psychiatry 26, 186-202 (2021).

25. Benedetti, F. et al. A glycogen synthase kinase 3-beta promoter gene single nucleotide polymorphism is associated with age at onset and response to total sleep deprivation in bipolar depression. Neurosci. Lett. 368, 123-126 (2004).

26. Benedetti, F. et al. A length polymorphism in the circadian clock gene Per3 influences age at onset of bipolar disorder. Neurosci. Lett. 2008 445, 184-187 (2008).

27. Bellivier, F. et al. Serotonin transporter gene polymorphism influences age at onset in patients with bipolar affective disorder. Neurosci. Lett. 334, 17-20 (2002).

28. Tang, S., Machaalani, R. \& Waters, K. A. Brain-derived neurotrophic factor (BDNF) and TrkB in the piglet brainstem after post-natal nicotine and intermittent hypercapnic hypoxia. Brain Res. 1232, 195-205 (2008).

29. Etain, B. et al. A SNAP25 promoter variant is associated with early-onset bipolar disorder and a high expression level in brain. Mol. Psychiatry 15, 748-755 (2010).

30. Faraone, S. V., Lasky-Su, J., Glatt, S. J., Van Eerdewegh, P. \& Tsuang, M. T. Early onset bipolar disorder: possible linkage to chromosome 9q34. Bipolar Disord. 8, 144-151 (2006).

31. Lin, P. I. et al. Assessment of the effect of age at onset on linkage to bipolar disorder: evidence on chromosomes 18p and 21q. Am. J. Hum. Genet. 77 545-555 (2005)

32. Grozeva, D. et al. Reduced burden of very large and rare CNVs in bipolar affective disorder. Bipolar Disord. 15, 893-898 (2013).

33. Kennedy, K. P., Cullen, K. R., DeYoung, C. G. \& Klimes-Dougan, B. The genetics of early-onset bipolar disorder: a systematic review. J. Affect. Disord. 184, 1-12 (2015).

34. Noor, A. et al. Copy number variant study of bipolar disorder in Canadian and UK populations implicates synaptic genes. Am. J. Med. Genet. B Neuropsychiatr. Genet. 165B, 303-313 (2014).

35. Priebe, L. et al. Genome-wide survey implicates the influence of copy number variants (CNVs) in the development of early-onset bipolar disorder. Mol. Psychiatry 17, 421-432 (2012).

36. Jamain, $\mathrm{S}$. et al. Common and rare variant analysis in early-onset bipolar disorder vulnerability. PLoS One 9, e104326 (2014).

37. Belmonte Mahon, P. et al. Genome-wide association analysis of age at onset and psychotic symptoms in bipolar disorder. Am. J. Med. Genet. B Neuropsychiatr. Genet. 156B, 370-378 (2011).

38. Chen, C. K. et al. GADL1 variant and medication adherence in predicting response to lithium maintenance treatment in bipolar I disorder. BJPsych Open 2, 301-306 (2016).

39. Lee, M. T. et al. Genome-wide association study of bipolar I disorder in the Han Chinese population. Mol. Psychiatry 16, 548-556 (2011).

40. Cheng, A. T. et al. Cross-cultural implementation of a Chinese version of the Schedules for Clinical Assessment in Neuropsychiatry (SCAN) in Taiwan. Br. J. Psychiatry 178, 567-572 (2001).

41. Denicoff, K. D. et al. Validation of the prospective NIMH-Life-Chart Method (NIMH-LCM-p) for longitudinal assessment of bipolar illness. Psychol. Med. 30 1391-1397 (2000). 
42. Singh, T. Pediatric bipolar disorder: diagnostic challenges in identifying symptoms and course of illness. Psychiatry (Edgmont) 5, 34-42 (2008).

43. Arango, C., Fraguas, D. \& Parellada, M. Differential neurodevelopmental trajectories in patients with early-onset bipolar and schizophrenia disorders. Schizophr. Bull. 40, S138-S146 (2014).

44. 1000 Genomes Project Consortium, et al. A global reference for human genetic variation. Nature 526, 68-74 (2015).

45. Wang, K., Li, M. \& Hakonarson, H. ANNOVAR: functional annotation of genetic variants from high-throughput sequencing data. Nucleic Acids Res. 38, e164 (2010).

46. Purcell, S. et al. PLINK: a tool set for whole-genome association and population-based linkage analyses. Am. J. Hum. Genet. 81, 559-575 (2007).

47. Thomas, L. A., Akins, M. R. \& Biederer, T. Expression and adhesion profiles of SynCAM molecules indicate distinct neuronal functions. J. Comp. Neurol. 510, 47-67 (2008)

48. Clarke, T. K. et al. Genome-wide association study of alcohol consumption and genetic overlap with other health-related traits in UK Biobank $(N=112$ 117). Mol. Psychiatry 22, 1376-1384 (2017).

49. Pasman, J. A. et al. GWAS of lifetime cannabis use reveals new risk loci, genetic overlap with psychiatric traits, and a causal influence of schizophrenia. Nat. Neurosci. 21, 1161-1170 (2018).

50. Stringer, S. et al. Genome-wide association study of lifetime cannabis use based on a large meta-analytic sample of 32330 subjects from the International Cannabis Consortium. Transl. Psychiatry 6, e769 (2016).

51. Boutwell, B. et al. Replication and characterization of CADM2 and MSRA genes on human behavior. Heliyon 3, e00349 (2017).

52. Day, F. R. et al. Physical and neurobehavioral determinants of reproductive onset and success. Nat. Genet. 48, 617-623 (2016).
53. Ibrahim-Verbaas, C. A. et al. GWAS for executive function and processing speed suggests involvement of the CADM2 gene. Mol. Psychiatry 21, 189-197 (2016).

54. Davies, G. et al. Genetic contributions to variation in general cognitive function: a meta-analysis of genome-wide association studies in the CHARGE consortium $(N=53949)$. Mol. Psychiatry 20 183-192 (2015).

55. Davies, G. et al. Genome-wide association study of cognitive functions and educational attainment in UK Biobank $(N=112$ 151). Mol. Psychiatry 21, 758-767 (2016).

56. Kathleen Holmes, M. et al. Conceptualizing impulsivity and risk taking in bipolar disorder: importance of history of alcohol abuse. Bipolar Disord. 11, 33-40 (2009).

57. Morris, J. et al. Genetic variation in CADM2 as a link between psychological traits and obesity. Sci. Rep. 9, 7339 (2019).

58. Dickerson, F., Stallings, C., Origoni, A., Boronow, J. \& Yolken, R. Elevated serum levels of C-reactive protein are associated with mania symptoms in outpatients with bipolar disorder. Prog. Neuropsychopharmacol. Biol. Psychiatry 31, 952-955 (2007).

59. Strawbridge, R. J. et al. Genome-wide analysis of self-reported risk-taking behaviour and cross-disorder genetic correlations in the UK Biobank cohort. Transl. Psychiatry 8, 39 (2018).

60. Zhang, S., Zhang, H., Zhu, J., Zhang, X. \& Liu, Y. MiR-522 contributes to cell proliferation of human glioblastoma cells by suppressing PHLPP1 expression. Biomed. Pharmacother. 70, 164-169 (2015).

61. Egeland, J. A., Blumenthal, R. L., Nee, J., Sharpe, L. \& Endicott, J. Reliability and relationship of various ages of onset criteria for major affective disorder. J. Affect. Disord. 12, 159-165 (1987). 